\title{
EFICIÊNCIA DE INSETICIDAS À BASE DE NIM NO CONTROLE DE Grapholita molesta (BUSCK, 1916) (LEPIDOPTERA: TORTRICIDAE) ${ }^{1}$
}

\author{
ODERLEI BERNARDI ${ }^{2}$, DANIEL BERNARDI ${ }^{3}$, CALISC DE OLIVEIRA TRECHA $^{4}$, \\ EDSON DE OLIVEIRA JARDIM ${ }^{5}$, UEMERSON SILVA DA CUNHA ${ }^{6}$, MAURO SILVEIRA GARCIA ${ }^{7}$
}

RESUMO - O objetivo foi avaliar a eficiência de inseticidas à base de nim no controle da mariposa-oriental G. molesta em laboratório. A dieta artificial foi cortada em cubos e imersa nas caldas dos respectivos tratamentos; em seguida, lagartas recém-eclodidas foram transferidas para tubos de vidro, contendo os cubos de dieta tratados. Os produtos testados foram: NeemAzal-T/S ${ }^{\circledR}$, Dalneem emulsionável ${ }^{\circledR}$, Organic Neem $^{\circledR}$ e Natuneem Agrícola ${ }^{\circledR}$ na concentração de $0,5 \%$ do produto comercial (p.c.) em comparação com o inseticida químico fenitrotion (Sumithion $500 \mathrm{CE}^{\circledR}$ ) a $0,15 \%$ p.c. e uma testemunha (água). Os inseticidas NeemAzal-T/ $\mathrm{S}^{\circledR}$ e Dalneem emulsionável ${ }^{\circledR}$ apresentaram a mortalidade de $100 \%$ das lagartas, sendo eficientes no controle do inseto em condições de laboratório. Organic $\mathrm{Neem}^{\circledR}$ e Natuneem Agrícola ${ }^{\circledR}$ demonstraram menor atividade inseticida, ocasionando mortalidade de 73,5\% e 22,5\%, respectivamente; no entanto, afetaram significativamente a viabilidade larval.

Termos para indexação: Azadiractha indica, nim, mariposa-oriental.

\section{EFFICIENCY OF NEEM INSECTICIDES IN THE CONTROL OF Grapholita molesta (BUSCK, 1916) (LEPIDOPTERA: TORTRICIDAE)}

\begin{abstract}
The objective was to evaluate the efficiency of neem insecticides in the control of oriental fruit moth Grapholita molesta in laboratory conditions. The artificial diet was cut in cubes and immersed in the syrups of the respective treatments; soon afterwards, recently-emerged caterpillars were transferred, for glass tubes, containing the diet cubes treated. The tested products were: NeemAzal-T/S ${ }^{\circledR}$, Dalneem emulsifiable $^{\circledR}$, Organic Neem ${ }^{\circledR}$ and Natuneem Agricultural ${ }^{\circledR}$ in the concentration of $0.5 \%$ of the commercial product (p.c.) compared with the chemical insecticide fenitrotion (Sumithion $500 \mathrm{CE}^{\circledR}$ ) to $0.15 \%$ p.c. and a witness (water). The insecticides NeemAzal-T/S ${ }^{\circledR}$ and Dalneem emulsifiable ${ }^{\circledR}$ presented a mortality of $100 \%$ of the caterpillars being efficient in the control of the insect in laboratory conditions. Organic $\mathrm{Neem}^{\circledR}$ and Natuneem Agricultural $^{\circledR}$ demonstrated lower insecticidal activity, causing mortality of $73.5 \%$ and $22.5 \%$ respectively, however, significantly affected the larval viability.
\end{abstract}

Index terms: Azadiractha indica, neem, oriental fruit moth.

Grapholita molesta (Busck, 1916) (Lepidoptera: Tortricidae), conhecida comumente por mariposa-oriental ou broca-dos-ponteiros, é considerada atualmente uma das principais pragas do pessegueiro e de outras frutíferas de clima temperado (Botton et al., 2001).

Os danos provocados pelo inseto, observados tanto nas brotações do ano como nos frutos, são ocasionados exclusivamente pelas lagartas, que, em geral, perfuram o ponteiro principal, ramos secundários, pecíolos e frutos (Salles, 1991). Nos frutos, os maiores danos ocorrem na fase de endurecimento do caroço (Poltronieri et al., 2008).

No controle do inseto, basicamente são utilizados inseticidas químicos, muitas vezes de amplo espectro e altamente tóxicos. Diante da necessidade de adoção de táticas menos impactantes de controle, os extratos de plantas vêm sendo estudados como

'(Trabalho 013-09). Recebido em: 07-01-2009. Aceito para publicação em: 23-10-2009.

${ }^{2}$ Eng. Agr., Doutorando do Depto. de Entomologia, Fitopatologia e Zoologia Agrícola/ESALQ/USP, Av. Pádua Dias, 11, CEP: 13418900, Piracicaba-SP, oderleibernardi@yahoo.com.br

${ }^{3}$ Eng. Agr., Depto. de Fitossanidade/FAEM/UFPel, C.P. 354, CEP: 96010-900, Pelotas-RS. dbernardi2004@yahoo.com.br ${ }^{4}$ Estudante de Agronomia, Depto. de Fitossanidade/FAEM/UFPel, C. P. 354, CEP: 96010-900, Pelotas-RS, calisc_oliveiratrecha@yahoo.com.br ${ }_{5}^{5}$ Estudante de Agronomia, Depto. de Fitossanidade/FAEM/UFPel, C. P. 354, CEP: 96010-900, Pelotas-RS, eojardim@yahoo.com.br ${ }^{6}$ Eng. Agr., Dr., Prof. Depto. de Fitossanidade/FAEM/UFPel, C. P. 354, CEP: 96010-900, Pelotas-RS, uscunha@yahoo.com.br ${ }^{7}$ Eng. Agr., Dr., Prof. Depto. de Fitossanidade/FAEM/UFPel, C. P. 354, CEP: 96010-900, Pelotas-RS, garciasmauro@yahoo.com.br 
uma alternativa ao uso de inseticidas sintéticos. Dentre as diferentes espécies vegetais com potencial para o controle de pragas, destacam-se Azadiractha indica, popularmente denominada nim ou nime, como alternativa ao controle químico de diversos insetos-praga (Martinez, 2002), cujos extratos têm-se revelado tão potentes quanto os inseticidas organossintéticos comerciais (Schmutterer, 1990).

Nesse sentido, objetivou-se avaliar a eficiência de inseticidas à base de nim no controle da mariposa-oriental G. molesta em laboratório.

$\mathrm{O}$ experimento foi desenvolvido no Laboratório de Biologia de Insetos da Faculdade de Agronomia "Eliseu Maciel" (FAEM), Universidade Federal de Pelotas (UFPel), à temperatura de $25 \pm$ $1^{\circ} \mathrm{C}$, umidade relativa de $70 \pm 10 \%$ e fotofase de 16 horas.

A dieta artificial utilizada, composta à base de farinha de milho (Ariolli, 2007), após o preparo, foi vertida em caixas de plástico $\left(\right.$ Gerbox $\left.^{\circledR}\right)$, e posteriormente cortada em cubos de 1,0 cm de lado, os quais foram imersos nas caldas dos respectivos tratamentos por 3 segundos. Antes de utilizá-los, os cubos permaneceram em bandeja plástica, por 2 minutos, para o escorrimento do excesso de calda. Posteriormente, lagartas de G. molesta recémeclodidas foram transferidas, com o auxílio de um pincel fino, em câmara asséptica, para tubos de vidro $(2,5 \times 8,5 \mathrm{~cm})$, previamente esterilizados e tampados com algodão hidrófobo, contendo os cubos de dieta tratados.

Os produtos formulados à base de nim avaliados no bioensaio foram: NeemAzal-T/S ${ }^{\circledR}$, Dalneem emulsionável $^{\circledR}$, Organic Neem ${ }^{\circledR}$ e Natuneem Agríco$1 a^{\circledR}$ na concentração de $0,5 \%$ do produto comercial (p.c.). Como padrão de comparação, utilizou-se o inseticida químico fenitrotion (Sumithion $500 \mathrm{CE}^{\circledR}$ ) a $0,15 \%$ p.c. e uma testemunha com água.

Consideraram-se as seguintes variáveis: mortalidade diária até 12 dias após a inoculação (DAI), duração (dias) e viabilidade (\%) da fase larval.

O delineamento experimental foi o inteiramente casualizado, com 10 repetições, cada uma com quatro tubos (três lagartas/tubo), totalizando 120 indivíduos por tratamento. A análise estatística foi executada pelo programa "SANEST" (Zonta \& Machado, 1984), sendo as médias comparadas pelo teste de Tukey $(\mathrm{P} \leq 0,05)$. Com base no teste de Hartley, que avalia homogeneidade de variâncias, não se fez transformação de dados.

Foi verificada uma variação acentuada na mortalidade promovida pelos inseticidas. Dentre os produtos testados, destacam-se NeemAzal-T/S ${ }^{\circledR}$ e
Dalneem emulsionável ${ }^{\circledR}$, como os mais eficientes, causando mortalidade de $100 \%$ das lagartas, não diferindo significativamente do inseticida Sumithion $500 \mathrm{CE}^{\circledR}$, tomado como padrão de comparação (Tabela 1). Por outro lado, os inseticidas Organic $\mathrm{Neem}^{\circledR}$ e Natuneem Agrícola ${ }^{\circledR}$, com eficiência de controle de 73,2 e $22,5 \%$, respectivamente, embora inferiores à do padrão, superaram significativamente o tratamento-testemunha (Tabela 1).

A eficiência de NeemAzal-T/S ${ }^{\circledR}$ no controle de diversos insetos-praga já foi relatada. Silva et al. (2003) observaram a eficiência desse produto no controle de adultos de Bemisia argentifolli (Bellows \& Perring) (Hemiptera: Aleyrodidae), tanto em condição de casa de vegetação como a campo. Em casa de vegetação, aos 14 dias após pulverização (DAP), o controle dos insetos foi de $84,78 \%$ e $59,86 \%$ nas concentrações de $32 \mathrm{~mL}$ e $16 \mathrm{~mL}$ do p.c. $\mathrm{L}^{-1}$ de calda, respectivamente. Já a campo, aos 7 DAP, verificaram $67,83 \%$ e 70,13\% de eficiência com 4,0 mL e 8,0 mL do p.c. $\mathrm{L}^{-1}$ de calda, respectivamente.

Barros et al. (2005) constataram que NeemAzal-T/S ${ }^{\circledR}(1,0 \%)$, a campo, controlou eficientemente Frankliniella schultzei (Trybom) (Thysanoptera: Thripidae) na cultura do algodoeiro, com mortalidade de $85,71 \%$ aos 3 DAP, não diferindo do produto acetamiprid (Saurus), padrão de comparação. Entretanto, os produtos Nim-I-Go ${ }^{\circledR}$ e NimKol LS ${ }^{\circledR}$ ambos a 1,0\%, mostraram-se eficientes somente aos 7 DAP, ocasionando mortalidade de $70 \%$ dos insetos.

No presente trabalho, verificou-se também que o produto NeemAzal-T/S ${ }^{\circledR}$ demonstrou toxicidade semelhante ao inseticida-padrão, ocasionando mortalidade de $100 \%$ dos insetos aos 3 dias após a inoculação (DAI) (Figura 1). Essa eficiência, possivelmente, esteja relacionada à concentração definida de azadiractina presente nesse produto, o que também pode ter influenciado o eficiente controle de $G$. molesta, quando comparado aos demais inseticidas avaliados, nos quais a concentração de azadiractina não é especificada.

Para G. molesta, a mortalidade de lagartas até 3 DAI com NeemAzal-T/S ${ }^{\circledR}$ demonstra excelente potencial deste produto, principalmente pelo hábito de penetrar em frutos e ponteiros, exigindo controle já nas fases iniciais do seu desenvolvimento. A mortalidade de lagartas nos primeiros instares por produtos à base de nim foi relatada por Rodríguez \& Vendramim (1997), para lagartas de S. frugiperda criadas em dieta artificial com extrato de sementes de nim (5\%). A eficiência de produtos à base de nim é destacada por Góez et al. (2003), que constataram que dentre vários extratos vegetais, aqueles oriundos de $A$. indica, estão entre os mais eficientes no controle 
de diversos lepidópteros-praga.

Para os produtos Organic Neem ${ }^{\circledR}$ e Natuneem Agrícola ${ }^{\circledR}$, registrou-se mortalidade até 12 DAI (Figura 1). Aos 12 DAI, Dalneem emulsionável ${ }^{\circledR}$ mostrou-se tão eficiente quanto NeemAzal-T/S $\mathrm{S}^{\circledR}$ e o inseticida-padrão. Já o Natuneem Agrícola ${ }^{\circledR}$ demonstrou efeito tóxico pronunciado apenas até $3 \mathrm{DAI}$, e os insetos após este período foram pouco afetados pelo produto (Figura 1). Tal constatação pode estar relacionada à degradação dos compostos com atividade inseticida.

A duração da fase larval dos insetos, cuja dieta continha Natuneem Agrícola ${ }^{\circledR}$ não diferiu significantemente em relação à testemunha. No entanto, a viabilidade das lagartas foi drasticamente reduzida $(54,1 \%)$ em comparação à testemunha $(97,3 \%)$ (Tabela 1), o que sugere um efeito fagodeterrente ou fisiológico sobre os insetos. Mordue (Luntz) \& Nisbet (2000) consideram os efeitos fisiológicos dos extratos de nim como a interferência no crescimento, nos processos de metamorfose, na reprodução e em outros processos celulares muito mais consistentes que os de inibição alimentar.
Para a cultura do pessegueiro, além da grafolita, outros insetos podem sofrer efeitos de produtos à base de nim, como relatado por Salles \& Rech (1999), que verificaram que extratos de nim (A. indica) e cinamomo (Melia azedarach L.) têm ação inseticida por meio da redução da oviposição e no desenvolvimento de larvas e pupas da mosca-dasfrutas Anastrepha fraterculus (Wiedemann) (Diptera: Tephritidae).

O emprego de inseticidas naturais, sobretudo à base de nim, é uma boa opção para o manejo integrado de pragas, podendo vir a contribuir para a redução do uso de inseticidas mais agressivos aos organismos benéficos e ao ambiente. Além disso, segundo Mordue, Luntz e Nisbet (2000), existe uma larga margem de segurança para o emprego do nim, tanto para o usuário (produtor) quanto para o consumidor.

Os produtos NeemAzal-T/S ${ }^{\circledR}$ e Dalneem emulsionável $^{\circledR}$, aplicados a $0,5 \%$ (p.c.) em dieta artificial, são eficientes para o controle de lagartas da mariposa-oriental G. molesta em laboratório.

TABELA 1 - Mortalidade de lagartas de Grapholita molesta alimentadas com dieta artificial tratada com inseticidas á base de nim, aos 12 dias após a aplicação dos tratamentos e efeito na duração e viabilidade da fase larval.

\begin{tabular}{|c|c|c|c|}
\hline \multirow{2}{*}{ Tratamento (\% p.c.) } & \multirow{2}{*}{ Mortalidade (\%) } & \multicolumn{2}{|c|}{ Fase larval } \\
\hline & & Duração (dias) & Viabilidade (\%) \\
\hline Sumithion $500 \mathrm{CE}^{\circledR}(0,15)$ & $100,0 \mathrm{a}$ & - & $0 \mathrm{a}$ \\
\hline NeemAzal - T/S ${ }^{\circledR}(0,5)$ & $100,0 \mathrm{a}$ & - & $0 \mathrm{a}$ \\
\hline Dalneem emulsionável ${ }^{\circledR}(0,5)$ & $100,0 \mathrm{a}$ & - & $0 \mathrm{a}$ \\
\hline Organic Neem ${ }^{\circledR}(0,5)$ & $73,2 \mathrm{~b}$ & - & $5,0 \mathrm{a}$ \\
\hline Natuneem Agrícola $^{\circledR}(0,5)$ & $22,5 \quad \mathrm{c}$ & $15,7^{\mathrm{ns}}$ & $54,1 \quad$ b \\
\hline Testemunha-Água & 3,3 & 15,4 & $97,3 \quad \mathrm{c}$ \\
\hline $\mathrm{CV}(\%)$ & 11,8 & - & 19,1 \\
\hline
\end{tabular}

Médias seguidas por letras distintas diferem entre si, pelo teste de Tukey $(\mathrm{P} \leq 0,05)$

ns= não significativo

p.c.: produto comercial 


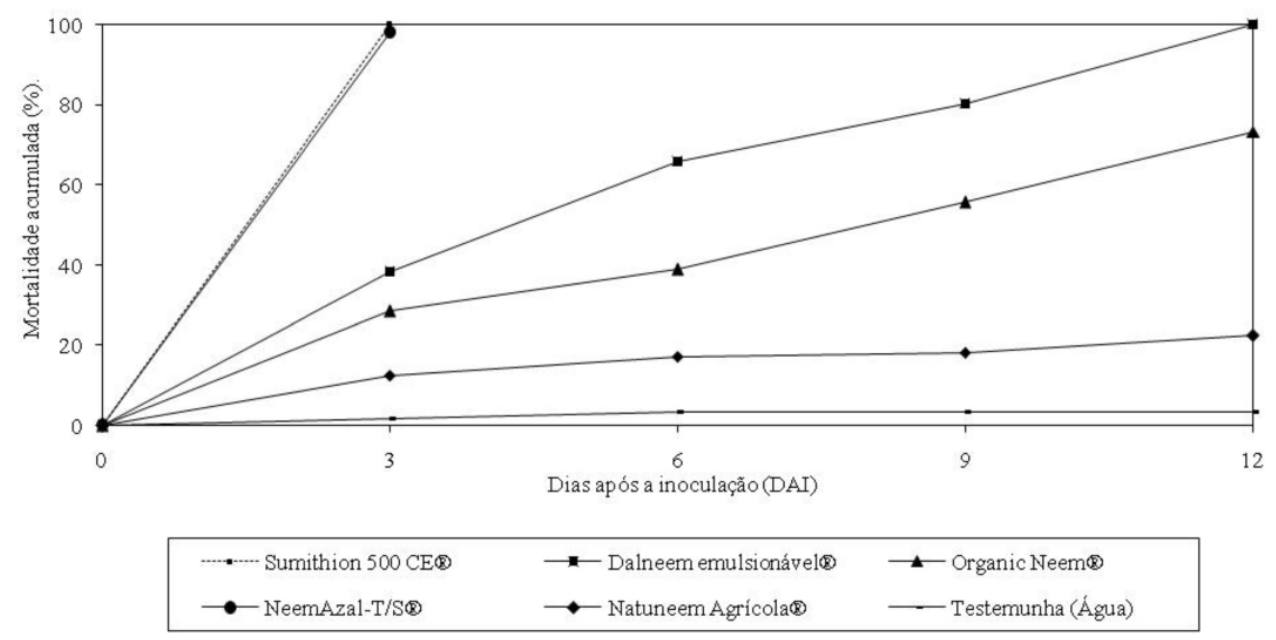

FIGURA 1- Mortalidade acumulada de Grapholita molesta (\%) em função do tempo de exposição aos diferentes inseticidas à base de nim, até 12 dias após a aplicação dos tratamentos.

\section{REFERÊNCIAS}

ARIOLLI, C. J. Técnica de criação e controle de Grapholita molesta (Busck) na cultura da macieira. 2007. 80f. Tese (Doutorado) - Faculdade de Agronomia "Eliseu Maciel", Universidade Federal de Pelotas, Pelotas, 2007.

BARROS, R. G.; OLIVEIRA, F. S. T. de; CZEPAK, C. Controle de Frankliniella schultzei na cultura do algodoeiro com aplicação de produtos formulados com Azadirachta indica. In: CONGRESSO BRASILEIRO DE ALGODÃO, 5., 2005. Campina Grande. Resumos.... Embrapa Algodão, 2005. 2p. Disponível em: <http://www.cnpa.embrapa.br/produtos/algodao/publicacoes/trabalhos/cba5/index/ html>. Acesso em: dia mês ano

BOTTON, M.; ARIOLli, C. J.; COLlETTA, V. D. Monitoramento da mariposa-oriental, Grapholita molesta (Busck, 1916), na cultura do pessegueiro. Bento Gonçalves: Embrapa Uva e Vinho, 2001. p.1-4. (Comunicado Técnico, 38). Disponível em: $<$ http://www.cnpuv.embrapa.br/publica/ comunicado/\#a2001>. Acesso em: dia mês ano

GÓEZ, G. B.; NERI, D. K. P.; CHAVES, J. W. N.; MARACAJÁ, P. B. Efeitos de extratos vegetais no controle de Spodoptera frugiperda (J.E. Smith) (Lepidoptera: Noctuidae). Caatinga, Mossoró, v.16, n.1/2, p.47-49, 2003.
MARTINEZ, S. S. O NIM: natureza, usos múltiplos, produção. Londrina: IAPAR, 2002. 142p.

MORDUE (LUNTZ), A. J.; NISBET, A. Azadirachtin from the neem tree Azadirachta indica: its actions against insects. Anais da Sociedade Entomológica do Brasil, Londrina, v.29, n.4, p.615-632, 2000.

POLTRONIERI, A. S.; SCHUBER, J. M.; MONTEIRO, L. B.; DE MIO, L. L. M. Danos de Grapholita molesta (Busck) (Lepidoptera: Tortricidae) em seis cultivares de pessegueiro, em Araucária, Paraná. Revista Brasileira de Fruticultura, Jaboticabal, v.30, n.4, p.897-901, 2008.

RODRÍGUEZ, H. C.; VENDRAMIM, J. D. Avaliação da bioatividade de extratos aquosos de Meliaceae sobre Spodoptera frugiperda. Revista de Agricultura, Piracicaba, v.72, n.3, p.305-318, 1997.

SALLES, L. A. B. Grafolita (Grapholita molesta): bioecologia e controle. Pelotas: Embrapa -Centro de Pesquisa Agropecuária de Clima Temperado, 1991. 14p. Disponível em: <http://www.cpact.embrapa.br/ publicacoes/catalogo/tipo/online $>$. Acesso em: dia mes ano.

SALLES, L. A.; RECH, N. L. Efeito de extratos de Nim (Azadiractha indica) e Cinamomo (Melia azedarachta) sobre Anastrepha fraterculus (Wied.) (Diptera: Tephritidae). Revista Brasileira de Agrociência, Pelotas, v.5, n.3, p.225-227, 1999. 
SCHMUTTERER, H. Properties and potential of natural pesticides from the neem tree, Azadirachta indica. Annual Review of Entomology, Palo Alto, v.35, p.217-297, 1990.

SILVA, L. D.; BLEICHER, E.; ARAÚJO, A. C. Eficiência de azadiractina no controle de mosca-branca em meoleiro sob condições de casa de vegetação e campo. Horticultura Brasileira, Brasília, v.21, n.2, p.198-201, 2003.
ZONTA, E. P.; MACHADO, A. A. SANEST: Sistema de análise estatística. Pelotas: Departamento de Matemática Estatística e Computação,Universidade Federal de Pelotas, 1984. 1 Disquete. 